vention der einzige signifikante Prädiktor für die Verbesserung der Depression nach acht Wochen. Erstmals konnten damit in einem kontrollierten randomisierten Design Hinweise auf die Wirksamkeit einer klettertherapeutischen Intervention bei Patienten mit Depression erbracht werden.

\section{Fazit für die Praxis}

- Bewegung wirkt sich positiv auf die psychische Gesundheit aus.

_ Bei der Behandlung von Menschen mit Depression sollte körperliche Aktivität als Therapiebaustein miteinbezogen werden.

_ Therapeutisches Klettern und Bouldern vereinen viele Wirkfaktoren moderner Depressionsbehandlung.

- Klettertherapeuten brauchen neben der therapeutischen Qualifikation ein fundiertes Kletter- und Sicherungskönnen.

_ Weitere Studien zur Überprüfung der Effektivität und Effizienz von therapeutischem Klettern bei Depression sind wünschenswert.

\section{Literatur}

www.springermedizin.de/dnp

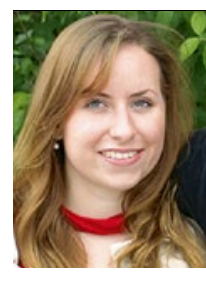

Stephanie Book, M. Sc. Psych.

Medizinische Psychologie und Med. Soziologie Psychiatrischen Universitätsklinik Erlangen (Leit. Prof. Dr. J. Kornhuber Friedrich-Alexander-Univ. Erlangen-Nürnberg Schwabachanlage 6 91054 Erlangen E-Mail: stephanie.book@ uk-erlangen.de

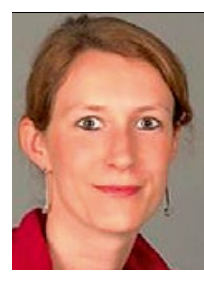

PD Dr. Katharina Luttenberger Dipl.-Psych. Medizinische Psychologie und Med. Soziologie Psychiatrischen Universitätsklinik Erlangen Friedrich-Alexander-Univ. Erlangen-Nürnberg Schwabachanlage 6 91054 Erlangen

\title{
Alterspsychotherapie und klinische Gerontopsychologie
}

Das vorliegende Buch, so der Herausgeber im Vorwort, verstehe sich als „Praktikerbuch für diejenigen, die sich entschieden haben, ältere Patienten psychotherapeutisch zu behandeln". Gegenüber der ersten Auflage sind unter Beibehaltung der drei Hauptkapitel "Grundlagen" und "Therapiemodelle", "Alterspsychotherapie" sowie "Klinische "Gerontopsychologie" Unterkapitel wie "Sucht", ",Somatoforme Störungen" oder auch „Palliative Medizin" hinzugekommen.

Noch immer spielt die psychotherapeutische Behandlung älterer Menschen entgegen der Bevölkerungsentwicklung und der damit verknüpften Zunahme psychischer Störungen im höheren Lebensalter eine erheblich vernachlässigte Rolle. Aus diesem Grund ist das vorliegende Buch allein schon ein wichtiger Beitrag zur Ermutigung und Befähigung psychotherapeutisch Arbeitender, sich dem älteren psychisch kranken Menschen verstärkt zu widmen und dabei, manch eingefahrenem Vorurteil zum Trotz, mit der Gewissheit unterwegs zu sein, in vielen Fällen spürbar und nachhaltig helfen zu können.

Das Buch stellt im ersten Hauptkapitel die Grundlagen und Therapiemodelle in nachvollziehbarer und teils erfreulich ausführlicher Weise dar; herauszuheben ist der US-amerikanische Beitrag über einen lebensspannenpsychologischen Ansatz im Alter mit integrativem Therapiekonzept. Der in diesem Hauptkapitel eingeordnete Rahmenbeitrag zur Psychopharmakologie ist paradigmatisch und informativ; angesichts der häufig notwendigen Kombinationsbehandlung von Psychotherapie mit Pharmakotherapie hätte der Beitrag auch ausführlicher gestaltet werden können. Dass hier die Neuroleptika lediglich als Untergruppe der Antidepressiva aufgeführt werden, ist wohl ein lektorales Versehen.

Im zweiten Hauptkapitel werden die großen Krankheitsfelder der Gerontopsychiatrie mit überwiegend ausführlich gestaltetem und vom interessierten Leser rasch verwertbarem Arbeitsmaterial dargelegt. Besonders gelungen sind hierbei die Klarheit der Wissensvermittlung und die Darstellung der jedem Thema eigenen wissenschaftlichen Evidenzstärke. Dieser Umstand erklärt, warum beispielsweise in den Unterkapiteln zu den mit relativ belastbaren Daten zur Psychotherapie versehenen Themen Depression und Angst ungleich mehr

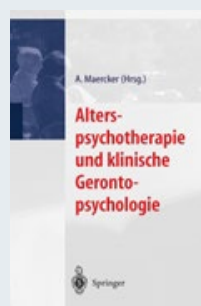

A. Maercker (Hrsg.)

Alterspsychotherapie und klinische Gerontopsychologie

Berlin Heidelberg: Springer-Verlag 2015, 2. Auflage, 390 Seiten, ISBN 978-3-642-54722-5, $49,99 €$

konkrete Arbeitsempfehlungen gegeben werden können als in noch wenig zur Psychotherapie wissenschaftlich erforschten Gebieten wie den sexuellen Dysfunktionen im Alter.

Einen starken Bezug zur institutionellen Versorgung erhielt das dritte Hauptkapitel, welches mit großem Gewinn für den in Senioren- oder Pflegeeinrichtungen tätigen Leser zahlreiche psychotherapeutische Hilfen und Informationsmaterialien bereitstellt. Diese beziehen sich auf diejenigen Interventionen, die etwa in der Pflege sowohl funktionsstabilisierend und progredienzvermeidend wirksam sind als auch etwa bei psychischen Einbußen autonomiestärkend und rückfallverhütend. Der abschließende, die palliative Medizin und Pflege behandelnde Teil stellt eine bezogen auf Schmerzsyndrome, irreversibel verlaufende Krankheitszustände und Sterbensprozesse für den Leser hilfreiche und wissensvertiefende Abrundung des Buches dar.

Für den etwas eiligeren, rasch den Überblick suchenden Leser sind die in den meisten Kapiteln zu findenden Zwischenzusammenfassungen von großem Nutzen; eine geringe Schwäche dieses Mehrautorenbuches könnte für ihn darin bestehen, dass er an mehreren Stellen des Buches mitunter inhaltsgleiche Informationen zu Erkrankungen finden kann. So sind beispielsweise in vier Kapiteln allgemeine Informationen zur Demenz enthalten. Hier wäre manchem Leser durch Einfügung von hilfreichen Querverweisen und der Abhandlung des Themas an einem Ort zu einer größeren Leseeffizienz verholfen.

Insgesamt ist die Lektüre des Buches allen in ärztlichen und psychologischen Berufen Tätigen zur Vertiefung ihres professionellen Wissens zu empfehlen, insbesondere aber denjenigen, die bereits erste Erfahrungen in der psychotherapeutischen Arbeit mit älteren Menschen gewonnen haben, um diese weiter zu vertiefen und perspektivisch zu komplettieren.

M. Haupt (Düsseldorf) 